\title{
Autorregulación del aprendizaje en la Formación Profesional a Distancia: efectos de la gestión del tiempo
}

\section{Self-regulated learning in Online Vocational Education and Training: effects of time management}

\author{
Cristian Jorge García-Marcos \\ Universidad de Sevilla. Sevilla, España \\ crigarmar9@alum.us.es \\ Omar López-Vargas \\ Universidad Pedagógica Nacional. Bogotá, Colombia \\ olopezv@pedagogica.edu.co \\ Julio Cabero-Almenara \\ Universidad de Sevilla. Sevilla, España \\ cabero@us.es
}

\section{Resumen}

La presente investigación estudia los efectos que dos herramientas relacionadas con la autorregulación del aprendizaje tienen sobre el logro académico, las conexiones por día y el tiempo de dedicación al estudio. Un total de 260 estudiantes de formación profesional cuyo aprendizaje se desarrolla en línea fueron asignados de forma aleatoria a tres grupos: un grupo interactuaba en un aula virtual a cuya estructura se le había añadido una herramienta que muestra una barra de progreso con las tareas a realizar durante el curso $(n=67)$; otro grupo interactuaba en un aula virtual al que se le añadía tanto la barra de progreso como una herramienta que estima el tiempo dedicación al estudio $(n=75)$; y el grupo de control interactuaba sin ninguna herramienta añadida con respecto a las que se trabaja habitualmente en el aula virtual $(\mathrm{n}=118)$. Se realizaron análisis factoriales ANOVA y MANOVA. Los resultados muestran que no existe una diferencia significativa en el logro académico entre grupos, independientemente del tipo del aula virtual en la que interactúen. Sin embargo, existen diferencias significativas en el tiempo de dedicación y en las conexiones diarias entre grupos, dependiendo del tipo de aula virtual empleado. Como conclusión, los estudiantes del grupo en el que se incluye la barra de progreso mejoran su eficiencia, ya que obtienen un logro académico similar al resto de estudiantes en aproximadamente la mitad de tiempo.

\section{Palabras clave}

tecnología educativa, formación profesional, aprendizaje en línea, autorregulación, gestión del tiempo

\begin{abstract}
The present research studies the effects that two tools related to self-regulated have on the academic achievement, the connections per day and the time of dedication to the study. A total of 260 vocational training e-learning students was randomly assigned to three groups: one group interacted in a virtual classroom in which a tool showing a progress bar with the tasks to be completed during the course was added $(n=67)$; another group interacted in a virtual classroom in which both the progress bar and a tool that estimates the dedication time to the study was added $(n=75)$; and the control group interacted without any extra tool added in the
\end{abstract}

RED. Revista de Educación a Distancia. Núm. 62, Vol. 20. Artíc. 06, 31-03-2020

DOI: http://dx.doi.org/10.6018/red.400071 
RED. Revista de Educación a Distancia. Núm. 62, Vol. 20. Artíc. 06, 31-03-2020

DOI: http://dx.doi.org/10.6018/red.400071

virtual classroom $(\mathrm{n}=118)$. ANOVA and MANOVA factor analysis were performed. The results show that there is no significant difference in academic achievement between groups, regardless of the type of the virtual classroom in which they interact. However, there are significant differences in the dedication time to the study and in the daily connections between groups, depending on the type of the virtual classroom was used. It is therefore concluded that the group in which the progress bar is included enhances efficiency, because students obtain similar academic achievements in approximately half the time.

\section{Keywords}

educational technology, vocational education and training, online learning, self-regulation, time management

\section{Introducción}

En la actualidad, los estudiantes que se forman bajo una modalidad de aprendizaje en línea -comúnmente conocida como e-learning- acceden a los materiales de estudio y realizan las diferentes actividades académicas en cualquier momento y en cualquier lugar, de una forma flexible y dinámica, atendiendo a las características de portabilidad y conectividad que les ofrecen las tecnologías de la información y la comunicación (TIC), mediante el uso de aulas virtuales (García-Peñalvo \& Seoane-Pardo, 2015; Gros, 2018).

Diversos autores afirman que el uso eficaz y eficiente de las aulas virtuales requiere de altas capacidades de autorregulación del aprendizaje por parte de los estudiantes, para que sean capaces de alcanzar los resultados deseados en su propio proceso de aprendizaje (Delen, Liew, \& Willson, 2014; Liaw \& Huang, 2013).

A su vez, existe cierto consenso entre la comunidad académica en proponer diversos factores que pueden estar asociados de una manera directa con la capacidad de autorregulación del aprendizaje de los estudiantes (Vohs \& Baumeister, 2016; Schunk \& Zimmermman, 2013). Entre estos factores se encuentra la denominada gestión del tiempo (Rowe \& Rafferty, 2013; Van Laer \& Elen, 2017). Si un estudiante no logra planificar y organizar bien su tiempo de estudio, es probable que deje para el último momento las tareas, que evidencie problemas de desmotivación, apatía para completar las actividades, bajos desempeños e incluso que, finalmente, aplace o abandone los estudios.

Ante esta problemática del aprendizaje en línea, algunos investigadores han propuesto el uso de herramientas de monitorización para fomentar entre los estudiantes el desarrollo de habilidades de gestión del tiempo de estudio. (Huertas, López-Vargas, \& SanabriaRodríguez, 2017; Valencia-Vallejo, López-Vargas, \& Sanabria-Rodríguez, 2018).

Autorregulación del aprendizaje en la Formación Profesional a Distancia: efectos de la gestión del tiempo Cristian Jorge García-Marcos, Omar López-Vargas y Julio Cabero Almenara.

Página 2 de 21 
RED. Revista de Educación a Distancia. Núm. 62, Vol. 20. Artíc. 06, 31-03-2020

DOI: http://dx.doi.org/10.6018/red.400071

Estas investigaciones se llevan a cabo principalmente en niveles universitarios, y es escaso el estudio de herramientas que incluyan estrategias pedagógicas y/o didácticas para favorecer la monitorización del proceso de aprendizaje en estudiantes en línea de nivel preuniversitario (Burrus, Jackson, Holtzman, Roberts, \& Mandigo, 2013; López-Vargas, Ibáñez-Ibáñez, \& Racines-Prada, 2017).

\subsection{Autorregulación del aprendizaje}

En general, un estudiante que autorregula su aprendizaje puede ser definido como una persona que es capaz de formularse metas de aprendizaje concretas, planear actividades para alcanzar dicha meta, monitorizar sistemáticamente su desempeño durante la ejecución de tales actividades, autoevaluarse de manera continua de acuerdo con las metas fijadas, efectuar los ajustes necesarios en función de la meta y, finalmente, valorar el resultado final de su aprendizaje (Pintrich, 2004; Zimmerman \& Schunk, 2011. Tradicionalmente, la autorregulación del aprendizaje supone la articulación de variables tanto motivacionales como cognitivas.

La autorregulación de la motivación implica el control activo de creencias motivacionales como: niveles altos de autoeficacia, una orientación de tipo intrínseco al dominio de conocimiento, la asignación de un elevado valor a la tarea de aprendizaje y una creencia alta para controlar el proceso de aprendizaje (Wolters, 2011; Schunk \& Zimmerman, 2012).

Respecto a las variables cognitivas, el estudiante autorregulado es capaz de usar diferentes estrategias de acuerdo con el contexto de la tarea y posee habilidades metacognitivas superiores para plantearse metas realistas, planificar la forma de conseguir dichas metas, monitorizar permanentemente su desempeño, evaluar de forma sistemática el estado actual de su aprendizaje para hacer los cambios o ajustes necesarios y, finalmente, reflexionar globalmente sobre el proceso a fin de optimizarlo en situaciones futuras (Hadwin, Järvelä, \& Miller, 2011; Zimmerman, 2013).

\subsection{Género y autorregulación del aprendizaje}

La manera en la que los estudiantes autorregulan su aprendizaje es objeto de estudio por múltiples investigadores (Panadero (2017), Parra, Cerda, López-Vargas \& Saiz (2014), Pintrich, 1995; Winne (2016)). Diferentes estudios muestran que tanto mujeres como hombres presentan diferencias individuales cuando deciden participar de forma activa en su propio proceso de aprendizaje (Basol \& Balgalmis, 2016; Bidjerano, 2005; Tseng, Liu, \& Nix, 2017).

Dentro de este ámbito de investigación, estudios como el de Fischer, Schult \& Hell (2013) presentan resultados en los que las mujeres tienen niveles de conciencia más altos que los hombres en lo referente al sentido del deber y la autodisciplina académica. En esta misma línea, Bidjerano (2005) encuentra que las mujeres tienen habilidades de autocontrol y que

Autorregulación del aprendizaje en la Formación Profesional a Distancia: efectos de la gestión del tiempo Cristian Jorge García-Marcos, Omar López-Vargas y Julio Cabero Almenara.

Página 3 de 21 
muestran altos niveles en la planificación del aprendizaje respecto a la formulación de metas y la selección de actividades para el logro de dichas metas en comparación con los hombres. En lo que concierne a la solicitud de ayuda social para el desarrollo de las tareas, se evidencia que las mujeres muestran una actitud positiva hacia el trabajo en grupo y hacia la petición de ayuda a los profesores para el desarrollo de las actividades de aprendizaje (Arroyo, Burleson, Tai, Muldner \& Woolf, 2013; Kessels \& Steinmayr, 2013; Yousaf, Popat \& Hunter, 2015). Sin embargo, otros estudios muestran que no existen diferencias significativas entre hombres y mujeres en relación a la gestión del esfuerzo y al uso de habilidades metacognitivas (Hederich-Martínez, Camargo-Uribe, \& López-Vargas, 2018; Studenska, 2011).

En cuanto al uso de las TIC, los estudios también muestran diferencias en el uso de las mismas por parte de hombres y mujeres. Por ejemplo, Tucker (2014) indica que los hombres son más propensos que las mujeres a realizar aportaciones en los foros. Por su parte, una investigación realizada por Rossing, Miller, Cecil, \& Stamper (2012) halla diferencias de género en el nivel de confort en relación al uso de la tecnología. De igual forma, en los estudios de Cooper, Schindler, \& Sun (2006), Imhof, Vollmeyer, \& Beierlein (2007) y Yuselturk \& Bulut (2009) también se evidencian mayores niveles de ansiedad en mujeres respecto a hombres cuando estos interactúan en un ambiente computacional.

\subsection{Gestión del tiempo en la autorregulación del aprendizaje en línea}

Frente a una determinada tarea, la planificación del aprendizaje consiste en que el estudiante defina sus metas académicas y tome decisiones de acción que resultan determinantes para el éxito en su proceso de aprendizaje como, por ejemplo, la elección de la estrategia, la selección de los materiales/recursos útiles durante el proceso y la definición de tiempos, entre otros. La planificación tiene entonces como insumo no solo un parámetro de logro, sino una serie de decisiones respecto a la manera en la que la meta debería de lograrse; establecidas las metas y los medios para alcanzarlas, el estudiante entra entonces en una etapa de realización de la tarea. Durante esta nueva etapa, la capacidad autorreguladora del individuo se pone de manifiesto en varios procesos importantes como el autocontrol, control del tiempo y monitorización (Rosário, Pereira, Högemann, Nunes, Figueiredo, Nuñez, Fuentes \& Gaeta, 2014, Hadwin, Järvelä, \& Miller, 2011).

El proceso de autocontrol requiere del estudiante una serie de estrategias para mantener la concentración y el interés durante su trabajo en la tarea. Con relación al factor tiempo, Rogers \& Mirra (2014) indican que aquello que un estudiante puede aprender depende del tiempo que dedique al aprendizaje. Si un estudiante es consciente de las metas académicas a alcanzar, entonces tratará de gestionar el tiempo de forma eficaz para lograrlas.

La monitorización corresponde a un proceso de auto-observación. Se trata en esencia de un seguimiento en paralelo de la actividad de la persona, durante el cual se toma nota de los

Autorregulación del aprendizaje en la Formación Profesional a Distancia: efectos de la gestión del tiempo Cristian Jorge García-Marcos, Omar López-Vargas y Julio Cabero Almenara.

Página 4 de 21 
RED. Revista de Educación a Distancia. Núm. 62, Vol. 20. Artíc. 06, 31-03-2020

DOI: http://dx.doi.org/10.6018/red.400071

resultados de aprendizaje alcanzados y del grado de éxito de las estrategias utilizadas. La monitorización lleva entonces un registro no solo del resultado logrado, sino de aquello que permitió ese resultado o impidió otros. Una monitorización sistemática de la gestión del tiempo para la entrega de las tareas constituye uno de los principales indicadores de un mayor logro académico en los estudiantes (Panadero \& Alonso-Tapia, 2014).

\subsection{Uso de herramientas de apoyo para la gestión del tiempo}

Una parte esencial de la presente investigación se basa en el uso de herramientas que permiten a los discentes monitorizar su tiempo de dedicación al estudio y su participación en cada una de las actividades que deben completar dentro de un periodo establecido.

En esta última década, ha aparecido un movimiento denominado Quantified-self, que podría traducirse como Cuantificación personal (Eynon, 2015; Lee, 2013; Rivera-Pelayo, Zacharias, Müller \& Braun, 2012). Este movimiento promueve la monitorización de datos que son relevantes en nuestras actividades diarias mediante el uso de la tecnología. Actualmente, la aplicación de esta tecnología se ha popularizado en ámbitos diferentes al de la educación, como es, por ejemplo, la salud (Sharon, 2017; Shin \& Biocca, 2017).

En base a la filosofía del movimiento Quantified-self, con el empleo de las herramientas de apoyo que van a ser utilizadas en el presente experimento, se suministra al estudiante un andamiaje para la adquisición de estrategias adecuadas que le permitan una mejor gestión de su tiempo de estudio, y así pueda realizar el conjunto de tareas establecidas y completar las actividades académicas con éxito en un tiempo determinado.

Se debe tener en cuenta que, tal como se indica en el estudio realizado por García \& Cabero (2016), Moodle es el ambiente de aprendizaje en línea -también conocido por las siglas EVA (de Entorno Virtual de Aprendizaje)- utilizado en las enseñanzas de la Formación Profesional bajo la modalidad semipresencial o a distancia (en adelante, FPaD) de toda la geografía española. Por esta razón, para el desarrollo del experimento se hace uso de dos herramientas (denominadas bloques en el vocabulario de Moodle) que se incorporaron dentro del EVA Moodle y se aplicaron como apoyo para favorecer la monitorización sistemática del proceso de aprendizaje.

\subsection{Preguntas de investigación}

La presente investigación tiene como objetivo determinar si el empleo de herramientas de monitorización en un EVA tiene efectos positivos sobre el logro académico, y si el género del estudiante puede estar asociado con dicho logro. De acuerdo con los planteamientos anteriormente expuestos, se proponen las siguientes preguntas de investigación:

Autorregulación del aprendizaje en la Formación Profesional a Distancia: efectos de la gestión del tiempo Cristian Jorge García-Marcos, Omar López-Vargas y Julio Cabero Almenara.

Página 5 de 21 
RED. Revista de Educación a Distancia. Núm. 62, Vol. 20. Artíc. 06, 31-03-2020

DOI: http://dx.doi.org/10.6018/red.400071

1. ¿Cuál es el efecto que genera sobre el logro académico en estudiantes de formación profesional el añadir en la estructura de un EVA herramientas de monitorización para gestionar su tiempo de estudio?

2. ¿Existen diferencias significativas en el logro académico entre hombres y mujeres de formación profesional que aprenden en línea?

3. ¿Existe una interacción significativa en el logro académico de acuerdo con el género del estudiante y el uso de herramientas de monitorización para gestionar el tiempo en el aprendizaje en línea?

4. ¿El tiempo de dedicación al estudio y las conexiones por día difieren entre estudiantes que aprenden con tipos de EVA en los que se incluyen en su estructura herramientas de monitorización para gestionar el tiempo, y los que aprenden con un tipo de EVA al que no se le incluye estas herramientas?

\section{Materiales y métodos}

\subsection{Diseño}

La investigación es de tipo experimental. Como variable independiente se toma el escenario de aula virtual, que tiene tres valores: grupo experimental 1, que interactúa en un aula virtual a cuya estructura se le ha añadido una herramienta que muestra una barra de progreso con las tareas a realizar durante el curso; grupo experimental 2, que interactúa en un aula virtual al que se le ha añadido tanto la barra de progreso como una herramienta que estima el tiempo dedicación al estudio; y grupo de control, que interactúa sin ninguna de estas herramientas. Existe una variable asociada, que es el género del estudiante. Las variables dependientes del estudio son tres: el logro académico, el tiempo de dedicación al módulo profesional y el número de conexiones por día.

\subsection{Muestra}

En el experimento participaron estudiantes del Institut Obert de Catalunya (IOC), centro educativo con sede en Barcelona desde el que se imparte la totalidad de la FPaD de Cataluña. Los participantes estaban matriculados en las titulaciones de Técnico Superior en Administración de Sistemas Informáticos en Red (ASIR), Técnico Superior en Desarrollo de Aplicaciones Multiplataforma (DAM) y Técnico Superior en Desarrollo de Aplicaciones Web (DAW) en las que cursaban los módulos profesionales de Implantación de Sistemas Operativos (ASIR)/Sistemas Informáticos (DAM y DAW), Gestión de Bases de Datos (ASIR), Bases de Datos (DAM y DAW) y Programación Básica (ASIR)/Programación (DAM). Del total de 444 participantes iniciales, no abandonaron el curso 260 estudiantes (59\%), con una edad que oscilaba entre 19 y 51 años ( $M=33$ años, DT=7), de los cuales 60

Autorregulación del aprendizaje en la Formación Profesional a Distancia: efectos de la gestión del tiempo Cristian Jorge García-Marcos, Omar López-Vargas y Julio Cabero Almenara.

Página 6 de 21 
RED. Revista de Educación a Distancia. Núm. 62, Vol. 20. Artíc. 06, 31-03-2020

DOI: http://dx.doi.org/10.6018/red.400071

eran mujeres y 200 hombres. Al finalizar el experimento, el número de estudiantes por grupo fue: 67 en el grupo experimental 1, 75 en el grupo experimental 2, y 118 en el grupo de control.

\subsection{Materiales}

\section{Bloque dedicación al curso}

Una de las dos herramientas para el EVA Moodle que se ha utilizado en la presente investigación es el bloque de "Dedicación al curso" (https://moodle.org/plugins/block_dedication) creado por Aday Talavera del Centro de Innovación para la Sociedad de la Información (CICEI) de la Universidad de las Palmas de Gran Canaria (ULPGC).

Esta herramienta permite visualizar una estimación del tiempo de dedicación al estudio (ver Imagen 1). La herramienta estaba desarrollada originalmente para que se mostrara únicamente al docente, por lo que se realizaron las modificaciones oportunas en el código fuente del bloque para que también pueda mostrarse a los estudiantes, de manera que se permitiera al docente designar en Moodle la visualización del bloque al grupo experimental correspondiente y ocultarlo al grupo de control.

\section{Imagen 1}

Vista del estudiante de la herramienta "Dedicación al curso" modificada ad-hoc para el experimento

\section{Dedicación al curso 미}

Tu tiempo de dedicación estimado es

11 horas 12 minutos

\section{Bloque barra de progreso}

La otra herramienta utilizada en la investigación es el bloque "Barra de progreso" (https://moodle.org/plugins/block_progress) desarrollado para el EVA Moodle por De Raadt \& Dekeyser (2009). Se trata de una herramienta que muestra de manera visual la información con la participación del estudiante en cada una de las actividades que debe realizar (ver Imagen 2). La herramienta utiliza una codificación de colores con la que el estudiante puede identificar las actividades que ya ha completado y las que todavía le quedan por realizar. Para el docente, la herramienta posee una vista general que le permite visualizar el progreso de todos los estudiantes de un grupo, con lo que esta herramienta

Autorregulación del aprendizaje en la Formación Profesional a Distancia: efectos de la gestión del tiempo Cristian Jorge García-Marcos, Omar López-Vargas y Julio Cabero Almenara. 
RED. Revista de Educación a Distancia. Núm. 62, Vol. 20. Artíc. 06, 31-03-2020

DOI: http://dx.doi.org/10.6018/red.400071

puede serle de ayuda para encontrar a estudiantes en riesgo, por ejemplo, de abandono escolar.

\section{Imagen 2}

Vistas, según el perfil, de la herramienta "Barra de progreso"

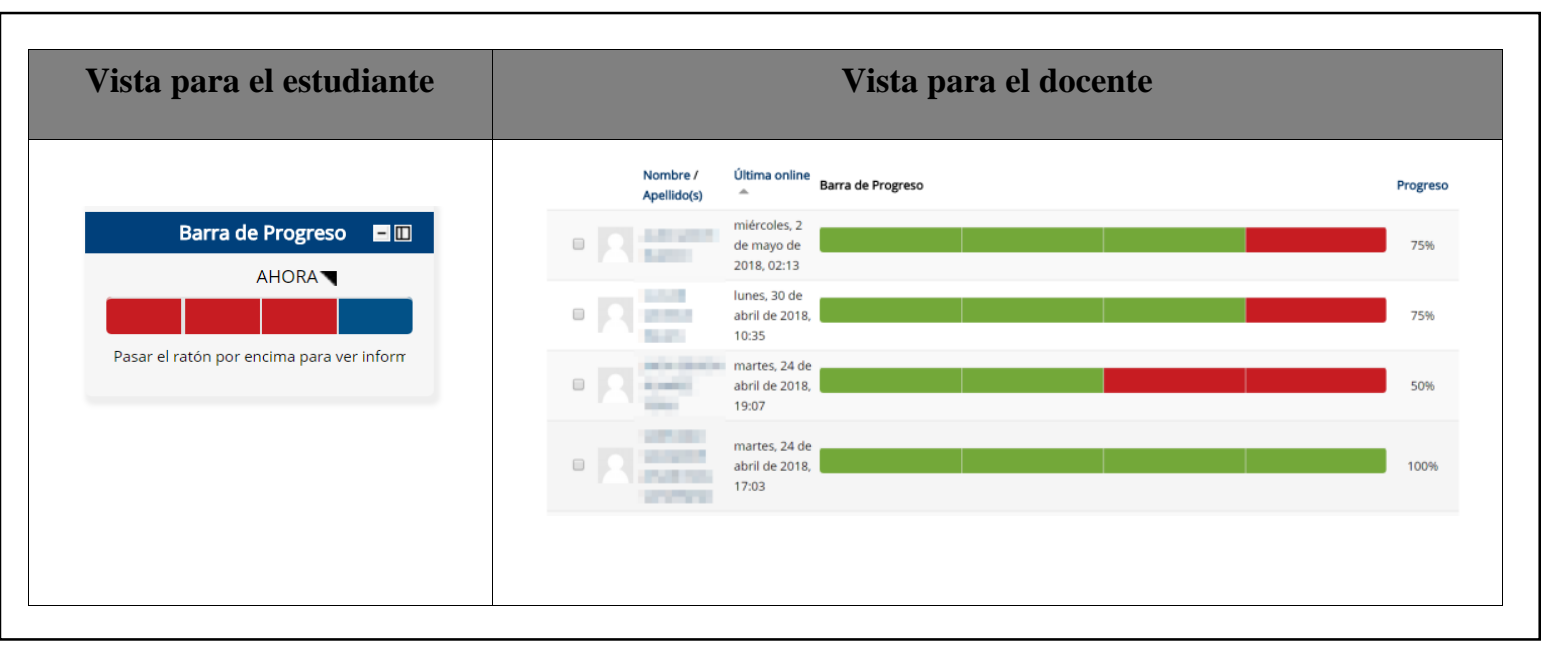

Autorregulación del aprendizaje en la Formación Profesional a Distancia: efectos de la gestión del tiempo Cristian Jorge García-Marcos, Omar López-Vargas y Julio Cabero Almenara. 
RED. Revista de Educación a Distancia. Núm. 62, Vol. 20. Artíc. 06, 31-03-2020

DOI: http://dx.doi.org/10.6018/red.400071

\section{Logro académico}

El logro académico viene dado por la calificación en el módulo profesional, que se calcula a través del promedio ponderado de las calificaciones obtenidas por el estudiante durante todo el semestre académico tanto en las pruebas prácticas como en las pruebas teóricas del módulo profesional. El número de pruebas por semestre varía según el módulo profesional, pero suele coincidir con una prueba cada quince días, es decir, se realizan evaluaciones quincenales para cada módulo profesional durante todo el semestre. La escala numérica utilizada en los estudios de Formación Profesional para la calificación de las notas es de un número entero comprendido entre 0 y 10.

\subsection{Procedimiento}

En primer lugar, se solicitó la autorización a los responsables del IOC en Barcelona, para que los estudiantes de las diferentes titulaciones participaran en la experimento. Tras la correspondiente autorización, se presentó la propuesta a los estudiantes y se les solicitó el consentimiento informado para participar en el estudio.

Posteriormente, se realizó una presentación a los docentes de las diferentes asignaturas para dar a conocer el funcionamiento de los dos bloques dentro del EVA.

La investigación es de tipo experimental, es decir, los participantes fueron asignados aleatoriamente a tres escenarios, que actuaban como variable independiente: 1) interacción con el EVA al que se le añadió el bloque barra de progreso con las actividades a realizar durante el curso; 2) interacción con el EVA al que se le añadió tanto el bloque barra de progreso como el bloque de dedicación al curso; y 3) interacción con el EVA al que no se le añadió ningún bloque adicional a los habituales del propio entorno. Los escenarios 1 y 2 corresponden a los grupos experimentales 1 y 2, respectivamente, y el escenario 3 corresponde al grupo de control. El experimento tenía el género del estudiante como variable asociada. Y las variables dependientes fueron tres: el logro académico, el tiempo de dedicación al estudio y el número de conexiones por día (estas dos últimas calculadas automáticamente por la herramienta de dedicación al curso).

El proceso de experimentación se desarrolló durante el segundo semestre, esto es, desde enero hasta junio. Durante este tiempo se hicieron comprobaciones periódicas de los registros en la base de datos. Una semana después de haber finalizado el periodo planificado para los módulos profesionales, el equipo técnico informático entregó la base de datos con los respectivos registros.

Previo al proceso de análisis, la base de datos se organizó y validó para garantizar la calidad de los datos, mediante el software Statistical Package for the Social Sciences (SPSS), versión 24.

Autorregulación del aprendizaje en la Formación Profesional a Distancia: efectos de la gestión del tiempo Cristian Jorge García-Marcos, Omar López-Vargas y Julio Cabero Almenara.

Página 9 de 21 


\section{Resultados}

\subsection{Efecto de los tipos de escenarios en el ambiente de aprendizaje en línea y el género del estudiante sobre el logro académico}

Se realizó un análisis factorial ANOVA 3x2. La variable dependiente del estudio fue el logro académico. Como variable independiente se tenía los escenarios en el ambiente en línea, con tres valores. El estudio tenía también una variable asociada, el género del estudiante. En la Tabla 1 se presenta el resumen de los estadísticos descriptivos de los grupos de estudiantes que trabajaron en cada escenario, teniendo en cuenta el género del estudiante. Conviene remarcar que únicamente se tuvieron en cuenta para el análisis los estudiantes que no abandonaron los módulos profesionales: de los 444 estudiantes que iniciaron el experimento, siguieron por completo el desarrollo del curso académico un total de 260 personas. Por tanto, el abandono de estudiantes en las titulaciones que participaron en el experimento fue del $58,6 \%$.

\section{Tabla 1}

Resultados del logro académico

\begin{tabular}{ccccccc}
\hline Grupo & \multicolumn{3}{c}{ Hombres } & \multicolumn{3}{c}{ Mujeres } \\
\cline { 2 - 7 } & $\begin{array}{c}\text { Calificación } \\
\text { media }\end{array}$ & DT & $\mathrm{N}$ & $\begin{array}{c}\text { Calificación } \\
\text { media }\end{array}$ & DT & $\mathrm{N}$ \\
\hline $\begin{array}{c}\text { Grupo de control } \\
\text { (sin bloques) }\end{array}$ & 6,51 & 3,226 & 53 & 6,21 & 2,992 & 14 \\
$\begin{array}{c}\text { Grupo experimental 1 } \\
\quad(1 \text { bloque) }\end{array}$ & 6,42 & 3,105 & 57 & 3,78 & 3,191 & 18 \\
$\begin{array}{c}\text { Grupo experimental 2 } \\
\text { (2 bloques) }\end{array}$ & 5,88 & 3,126 & 90 & 6,29 & 3,029 & 28 \\
\hline Total & 6,20 & 3,145 & 200 & 5,52 & 3,229 & 60 \\
\hline
\end{tabular}

A partir del análisis ANOVA se pudo evidenciar que existe una interacción significativa entre los factores principales del tipo de escenario utilizado en el EVA y el género del estudiante respecto al logro académico $\left[F(2,254)=4.065, p=.018, \eta^{2}=.031\right]$.

No obstante, no se halló un efecto principal significativo del tipo de escenario en el ambiente en línea $\left[F(2,254)=2.400, p=.093, \eta^{2}=.019\right]$ sobre el logro académico. A su vez, no existe un efecto principal significativo del género del estudiante $[F(1,254)=3.110$, $p=.071, \eta^{2}=.012$ ) sobre el logro académico. Sin embargo, el tamaño del efecto calculado

Autorregulación del aprendizaje en la Formación Profesional a Distancia: efectos de la gestión del tiempo Cristian Jorge García-Marcos, Omar López-Vargas y Julio Cabero Almenara.

Página 10 de 21 
para cada factor indica que cada uno de ellos explica una pequeña proporción de la varianza del logro académico.

La prueba post-hoc de Bonferroni indicó que los estudiantes masculinos que trabajaron con el tipo de escenario 1 en el EVA (es decir, con el bloque que muestra al estudiante una barra de progreso de las tareas a realizar durante el curso) obtuvieron mejores niveles de desempeño $(M=6.42, S D=3.105)$ comparados las mujeres $(M=3.78, S D=3.191)$ (ver Gráfico 1).

\section{Gráfico 1}

Efecto del tipo de escenario en el ambiente en línea y el género del estudiante sobre el logro académico

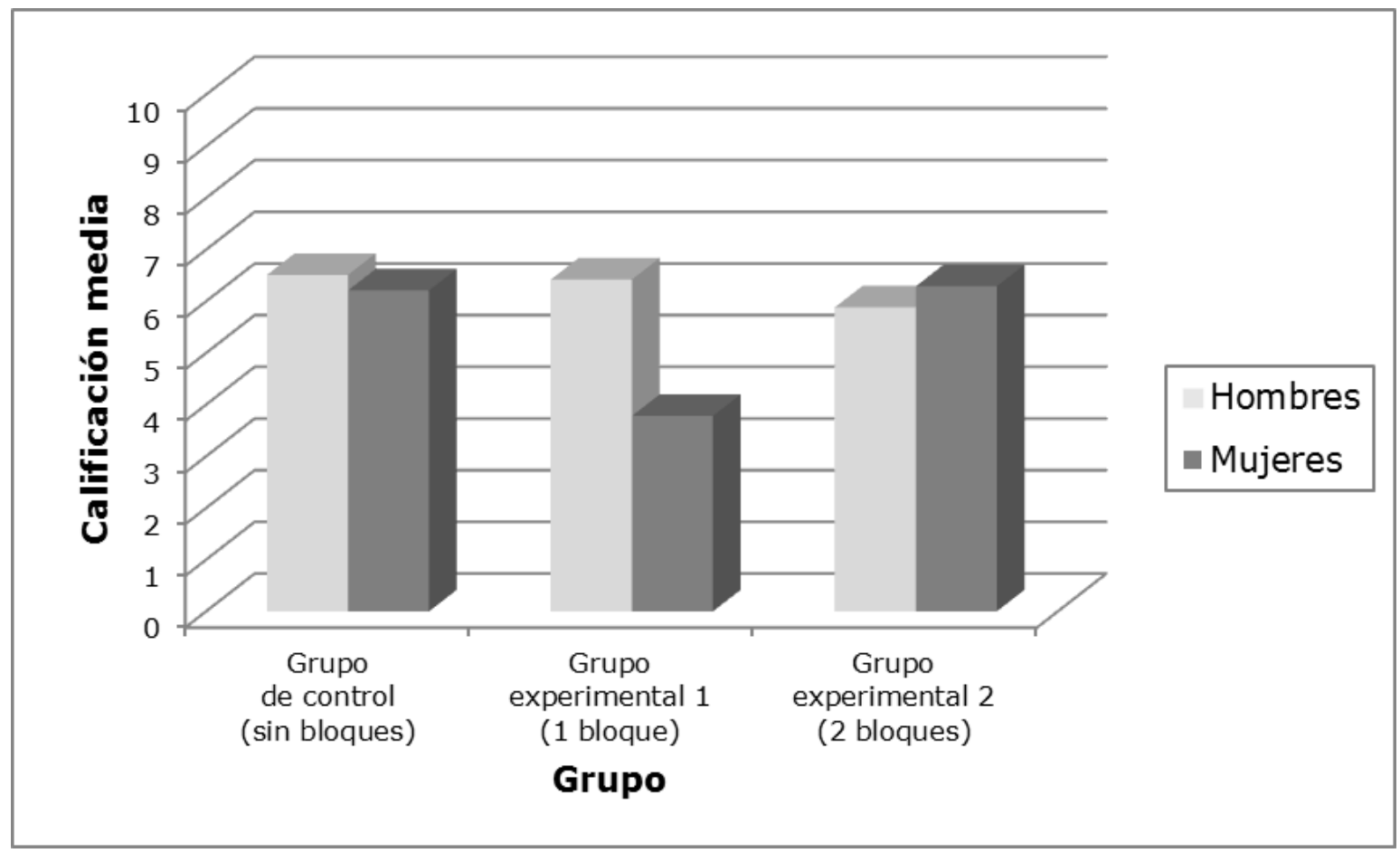

\subsection{Efecto del tipo de escenario en el ambiente de aprendizaje en línea sobre el tiempo de estudio y el número de conexiones}

Se realizó un análisis MANOVA. Las variables dependientes del estudio fueron el tiempo de dedicación al estudio y el número de conexiones por día. La variable independiente, es decir, el tipo de escenario empleado en el EVA, tenía tres valores. En la Tabla 2 se presenta el resumen de los estadísticos descriptivos.

Autorregulación del aprendizaje en la Formación Profesional a Distancia: efectos de la gestión del tiempo Cristian Jorge García-Marcos, Omar López-Vargas y Julio Cabero Almenara.

Página 11 de 21 
RED. Revista de Educación a Distancia. Núm. 62, Vol. 20. Artíc. 06, 31-03-2020

DOI: http://dx.doi.org/10.6018/red.400071

Tabla 2

Resultados de tiempo de dedicación al estudio y conexiones por día

\begin{tabular}{|c|c|c|c|c|c|}
\hline \multirow[t]{2}{*}{ Grupo } & \multicolumn{2}{|c|}{$\begin{array}{l}\text { Dedicación al estudio } \\
\text { (minutos) }\end{array}$} & \multicolumn{2}{|c|}{ Conexiones por día } & \multirow[t]{2}{*}{$\begin{array}{l}\text { Número de } \\
\text { participante }\end{array}$} \\
\hline & $\begin{array}{c}\text { Tiempo } \\
\text { medio }\end{array}$ & $\begin{array}{l}\text { Desviación } \\
\text { típica }\end{array}$ & $\begin{array}{c}\text { Tiempo } \\
\text { medio }\end{array}$ & $\begin{array}{l}\text { Desviación } \\
\text { típica }\end{array}$ & \\
\hline $\begin{array}{l}\text { Grupo de control } \\
\text { (sin bloques) }\end{array}$ & 1565,65 & 561,318 & ,6064 & ,13467 & 67 \\
\hline $\begin{array}{c}\text { Grupo experimental } 1 \\
\text { (1 bloque })\end{array}$ & 556,28 & 380,784 & ,4064 &, 15763 & 75 \\
\hline $\begin{array}{c}\text { Grupo experimental } 2 \\
\text { ( } 2 \text { bloques })\end{array}$ & 1468,69 & 681,307 & ,5797 &, 12170 & 118 \\
\hline
\end{tabular}

Los resultados de MANOVA indican que los diferentes escenarios en el EVA afectan significativamente las variables dependientes del estudio [Wilks ' $\Lambda=.626, F(4,510)=33.63$, $\left.p<.001, \eta^{2}=.209\right]$. Sin embargo, el tamaño de efecto multivariante es moderado.

Los resultados también indican que existen diferencias significativas en el tiempo de dedicación al estudio, dependiendo del escenario empleado en el EVA $[F(2,556)=72.15$, $\left.p<.001, \eta^{2}=.360\right]$. De igual manera, las conexiones diarias por parte de los estudiantes difieren significativamente $\left[F(2,556)=48.83, p<.001, \eta^{2}=.276\right]$.

Los resultados post-hoc de Tukey indican que los estudiantes que trabajaron con el escenario 1 en el EVA (es decir, con el bloque que muestra al estudiante una barra de progreso de las tareas a realizar durante el curso) obtuvieron un tiempo de dedicación menor $(M=556.28, S D=380.784)$ y difieren significativamente de los participantes que no utilizaron ningún bloque $(M=1556.65, S D=561.318)$ y quienes utilizaron dos bloques $(M=1468.69, S D=681.307)$.

No existen diferencias significativas entre el tiempo de dedicación para aquellos estudiantes que no utilizan bloques y quienes utilizan dos bloques.

De igual forma, los resultados post-hoc indican que los estudiantes que interactúan con un bloque tienen menos conexiones por día y difieren significativamente de aquellos estudiantes que trabajaron en los escenarios que no tienen ningún bloque o que tienen dos bloques (ver Gráfico 2).

Autorregulación del aprendizaje en la Formación Profesional a Distancia: efectos de la gestión del tiempo Cristian Jorge García-Marcos, Omar López-Vargas y Julio Cabero Almenara.

Página 12 de 21 
RED. Revista de Educación a Distancia. Núm. 62, Vol. 20. Artíc. 06, 31-03-2020

DOI: http://dx.doi.org/10.6018/red.400071

\section{Gráfico 2}

Efecto del tipo de escenario en el ambiente en línea sobre el tiempo de dedicación a la asignatura y sobre las conexiones por día
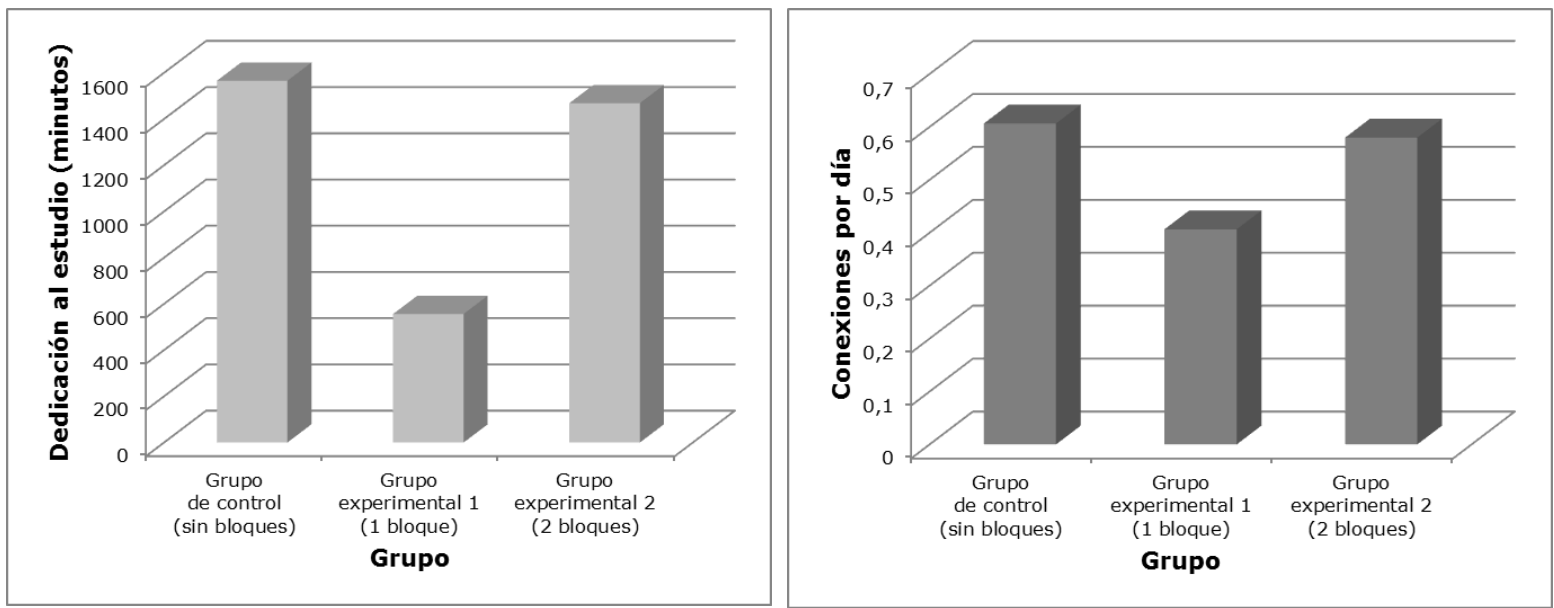

\section{Discusión y conclusiones}

En este artículo se ha descrito el experimento realizado a estudiantes de diferentes titulaciones de FPaD, con la finalidad de conocer cuáles son los efectos que la incorporación de herramientas de monitorización en un EVA tiene sobre el logro académico, y determinar si el género del estudiante está asociado con dicho desempeño. En concreto, las preguntas de investigación que se han propuesto son: 1. ¿Cuál es el efecto que genera sobre el logro académico en estudiantes de FPaD el añadir en la estructura de un EVA herramientas de monitorización para gestionar su tiempo de estudio?, 2. ¿Existen diferencias significativas en el logro académico entre hombres y mujeres de formación profesional que aprenden en línea?, 3. ¿Existe una interacción significativa en el logro académico de acuerdo con el género del estudiante y el uso de herramientas de monitorización para gestionar el tiempo en el aprendizaje en línea?, 4. ¿El tiempo de dedicación al estudio y las conexiones por día difieren entre estudiantes que aprenden con tipos de EVA en los que se incluyen en su estructura herramientas de monitorización para gestionar el tiempo, y los que aprenden con un tipo de EVA al que no se le incluye estas herramientas?

En referencia a la primera pregunta de investigación, los análisis del estudio indican que no existe un efecto relevante del tipo de escenario empleado en el EVA sobre el logro académico. Es decir, el hecho de incluir una o dos herramientas de monitorización para gestionar el tiempo de estudio, no ha supuesto una diferencia significativa en el logro académico de los estudiantes, ni de manera positiva ni negativa. Esto puede interpretarse de

Autorregulación del aprendizaje en la Formación Profesional a Distancia: efectos de la gestión del tiempo Cristian Jorge García-Marcos, Omar López-Vargas y Julio Cabero Almenara.

Página 13 de 21 
RED. Revista de Educación a Distancia. Núm. 62, Vol. 20. Artíc. 06, 31-03-2020

DOI: http://dx.doi.org/10.6018/red.400071

dos maneras: a) el empleo de las herramientas de apoyo utilizadas en el experimento no ha surtido el efecto de andamiaje sobre el estudiante para la adquisición de estrategias adecuadas que le permitan una mejor gestión de su tiempo de estudio y, por tanto, como el estudiante que recibe el andamiaje no ha adquirido habilidades de gestión de tiempo, tampoco mejora su rendimiento académico; o, b) si la inclusión de las herramientas de gestión del tiempo han servido para que el estudiante adquiera habilidades de gestión de tiempo, entonces se puede concluir que estos resultados coinciden con los de otras investigaciones, como las realizadas por Hamdan, Nasir, Khairudin, \& Sulaiman (2014) y Olowookere, Alao, Odukoya, Adekeye, \& Agbude (2015), en los que no se han hallado relaciones significativas entre habilidades de gestión del tiempo y logro académico.

En cuanto a la segunda pregunta de investigación, los datos indican que no existe un efecto principal significativo del género del estudiante de FPaD respecto al logro académico. Por tanto, se evidencia que los estudiantes, independientemente del género, obtienen similares resultados académicos, coincidiendo en este aspecto con las contribuciones de Amro, Mund, \& Kupczynski (2015) o Gambari, Shittu, Ogunlade, \& Osunlade (2017).

Por lo que respecta a la tercera pregunta de investigación, los resultados constatan que existe una interacción significativa entre los factores principales del tipo de escenario utilizado en el EVA y el género del estudiante respecto al logro académico. En concreto, si el estudiante es hombre, la media final obtenida en sus calificaciones no varía, independientemente del tipo de escenario EVA bajo el que haya aprendido. Sin embargo, si es mujer, el promedio alcanzado desciende de manera significativa en el caso de que el aprendizaje se haya desarrollado bajo el EVA en el que se incluye la barra de progreso, a pesar de que el promedio se mantiene en los mismos niveles que los hombres cuando el aprendizaje se desarrolla bajo los otros dos tipos de escenarios, es decir, sin bloques o con dos bloques incorporados al EVA. Estas diferencias están en línea con otras investigaciones que muestran diferencias en el género y el uso de los indicadores de autorregulación en el aprendizaje, y el uso de escenarios computacionales (Cooper et al., 2006; Imhof et al., 2007; Yuselturk \& Bulut, 2009).

Finalmente, en relación a la cuarta pregunta de investigación, los resultados muestran que los diferentes tipos de escenarios utilizados en el EVA afectan significativamente al tiempo de dedicación al estudio, así como también influyen en el número de conexiones diarias. En particular, los estudiantes que aprenden bajo el EVA que incluye una barra de progreso, registran un tiempo de dedicación al estudio significativamente menor a los que trabajan en los otros dos tipos de escenarios. Del mismo modo, los resultados indican que los estudiantes que interactúan con el EVA que incluye un único bloque emplean una cantidad de conexiones al día significativamente menor a aquellos que interactúan con los tipos de escenarios sin bloques o con dos bloques incorporados. Respecto a este asunto, se revela que la inserción de un único bloque en el EVA proporciona a los estudiantes el andamiaje

Autorregulación del aprendizaje en la Formación Profesional a Distancia: efectos de la gestión del tiempo Cristian Jorge García-Marcos, Omar López-Vargas y Julio Cabero Almenara.

Página 14 de 21 
necesario para la adquisición de habilidades de gestión del tiempo y, por ende, el tiempo de estudio se minimiza frente a los estudiantes que no han recibido andamiaje, ya que estos no han adquirido dichas habilidades. Sin embargo, también se puede observar que el tiempo de estudio se mantiene en valores similares entre los estudiantes que utilizan un andamiaje de dos bloques y aquellos que no poseen andamiaje. Este resultado, a priori contradictorio, podría deberse a que los estudiantes intentan obtener un tiempo de estudio similar al establecido en las recomendaciones de estudio proporcionadas por el profesor. Bajo esta perspectiva, el estudiante -sabedor de que tiene una herramienta de monitorización en la que se controla el tiempo de dedicación al estudio y esta también puede ser vista por el profesor- asemeja la duración de su estudio, de manera deliberada, a la duración recomendada en la planificación del módulo profesional.

A partir del desarrollo del experimento y de los resultados presentados, se puede concluir que el aprendizaje con la barra de progreso mejora la eficiencia académica, debido a que el estudiante obtiene el mismo logro académico aproximadamente en la mitad de tiempo. Para asentar los resultados de la presente investigación, es deseable continuar con el análisis de nuevas herramientas de apoyo que ayuden a los estudiantes a monitorizar ciertos aspectos de su proceso de aprendizaje, como medida de andamiaje para que puedan adquirir habilidades de gestión de su tiempo de estudio y que les permitan completar con éxito las tareas establecidas durante el desarrollo de su aprendizaje.

Presentación del artículo: 30 de septiembre de 2019

Fecha de aprobación: 26 de febrero de 2020

Fecha de publicación: 31 de marzo de 2020

García-Marcos, C.J., López-Vargas, O., y Cabero-Almenara, J. (2020). Autorregulación del aprendizaje en la Formación Profesional a Distancia: efectos de la gestión del tiempo. RED. Revista de Educación a Distancia, 20(62). DOI: http://dx.doi.org/10.6018/red.400071

\section{Financiación}

Este trabajo recibió el apoyo de una beca de movilidad entre universidades andaluzas e iberoamericanas otorgada por la Asociación Universitaria Iberoamericana de Postgrado (AUIP).

\section{Agradecimientos}

Nuestro agradecimiento a los estudiantes, técnicos y profesores del IOC por su colaboración en el desarrollo de la investigación.

Autorregulación del aprendizaje en la Formación Profesional a Distancia: efectos de la gestión del tiempo Cristian Jorge García-Marcos, Omar López-Vargas y Julio Cabero Almenara. 
RED. Revista de Educación a Distancia. Núm. 62, Vol. 20. Artíc. 06, 31-03-2020

DOI: http://dx.doi.org/10.6018/red.400071

\section{Referencias bibliográficas}

Amro, H., Mundy, M., \& Kupczynski, L. (2015). The effects of age and gender on student achievement in face-to-face and online college algebra classes. Research in Higher Education Journal, 27, 1-22. Recuperado de https://bit.ly/2KoBqbB

Arroyo, I., Burleson, W., Tai, M., Muldner, K., \& Woolf, B. (2013). Gender differences in the use and benefit of advanced learning technologies for mathematics. Journal of Educational Psychology, 105(4), 957-969. https://doi.org/10.1037/a0032748

Basol, G., \& Balgalmis, E. (2016). A multivariate investigation of gender differences in the number of online tests received-checking for perceived self-regulation. Computers in Human Behavior, 58, 388-397. https://doi.org/10.1016/j.chb.2016.01.010

Bidjerano, T. (2005). Gender Differences in Self-Regulated Learning. Documento presentado en el 36th Annual Meeting of the Northeastern Educational Research Association. Kerhonkson. Recuperado de https://bit.ly/2W9BxKY

Burrus, J., Jackson, T., Holtzman, S., Roberts, R., \& Mandigo, T. (2013). Examining the efficacy of a time management intervention for high school students. ETS Research Report Series, 2013(2), i-35. https://doi.org/10.1002/j.2333-8504.2013.tb02332.x

Cooper, D., Schindler, P., \& Sun, J. (2006). Business research methods (Vol. 9). New York: McGraw-Hill Irwin.

De Raadt, M., \& Dekeyser, S. (2009). A simple time-management tool for students' online learning activities. Documento presentado en el 26th Annual Conference of the Australian Society for Computers in Learning in Tertiary Education: Same Places, Different Spaces. Auckland. Recuperado de https://bit.ly/2WTYtC9

Delen, E., Liew, J., \& Willson, V. (2014). Effects of interactivity and instructional scaffolding on learning: Self-regulation in online video-based environments. Computers \& Education, 78, 312-320. https://doi.org/10.1016/j.compedu.2014.06.018

Eynon, R. (2015). The quantified self for learning: critical questions for education. Learning Media and Technology 40(4), 407-411. https://doi.org/10.1080/17439884.2015.1100797

Autorregulación del aprendizaje en la Formación Profesional a Distancia: efectos de la gestión del tiempo Cristian Jorge García-Marcos, Omar López-Vargas y Julio Cabero Almenara.

Página 16 de 21 
RED. Revista de Educación a Distancia. Núm. 62, Vol. 20. Artíc. 06, 31-03-2020

DOI: http://dx.doi.org/10.6018/red.400071

Fischer, F., Schult, J., \& Hell, B. (2013). Sex differences in secondary school success: why female students perform better. European Journal of Psychology of Education, 28(2), 529-543. https://doi.org/10.1007/s10212-012-0127-4

Gambari, A., Shittu, A., Ogunlade, O., \& Osunlade, O. (2017). Effectiveness of Blended Learning and Elearning Modes of Instruction on the Performance of Undergraduates in Kwara State, Nigeria. Malaysian Online Journal of Educational Sciences, 5(1), 25-36. Recuperado de https://bit.ly/2x8eh4V

García, C., \& Cabero, J. (2016). Evolución y estado actual del e-learning en la Formación Profesional española. RIED. Revista Iberoamericana de Educación a Distancia, 19(2), 167-191. https://doi.org/10.5944/ried.19.2.15800

García-Peñalvo, F., \& Seoane-Pardo, A. (2015). Una revisión actualizada del concepto de eLearning. Décimo Aniversario. Education in the knowledge society (EKS), 16(1), 119144. https://doi.org/10.14201/eks2015161119144

Gros, B. (2018). La evolución del e-learning: del aula virtual a la red. RIED. Revista Iberoamericana de Educación a Distancia, 21(2), 167-191. https://doi.org/10.5944/ried.21.2.20577

Hadwin, A. F., Järvelä, S., \& Miller, M. (2011). Self-regulated, co-regulated, and socially shared regulation of learning. En B. J. Zimmerman \& D. H. Schunk (eds.), Handbook of self-regulation of learning and performance (pp. 65-84). New York: Routledge.

Hamdan, A., Nasir, R., Khairudin, R., \& Sulaiman, W. (2014). Time management does not matter for academic achievement unless you can cope. International Proceedings of Economics Development and Research, 78, 22-26. Recuperado de https://bit.ly/2WFtQAf

Hederich-Martínez, C., Camargo-Uribe, A., \& López-Vargas, O. (2018). Motivation and use of learning strategies in students, men and women, with different level of schooling. Journal of Psychological and Educational Research, 26(1), 121-146. Recuperado de https://bit.ly/2wVcoIw

Huertas, A., López-Vargas, O., \& Sanabria-Rodríguez, L. (2017). Influence of a metacognitive scaffolding for information search in b-learning courses on learning achievement and its relationship with cognitive and learning style. Journal of $\begin{array}{llll}\text { educational computing } & \text { research, }\end{array}$ https://doi.org/10.1177/0735633116656634

Autorregulación del aprendizaje en la Formación Profesional a Distancia: efectos de la gestión del tiempo Cristian Jorge García-Marcos, Omar López-Vargas y Julio Cabero Almenara.

Página 17 de 21 
RED. Revista de Educación a Distancia. Núm. 62, Vol. 20. Artíc. 06, 31-03-2020

DOI: http://dx.doi.org/10.6018/red.400071

Imhof, M., Vollmeyer, R., \& Beierlein, C. (2007). Computer use and the gender gap: The issue of access, use, motivation, and performance. Computers in human behavior, 23(6), 2823-2837. https://doi.org/10.1016/j.chb.2006.05.007

Kessels, U., \& Steinmayr, R. (2013). Macho-man in school: Toward the role of gender role self-concepts and help seeking in school performance. Learning and Individual Differences, 23, 234-240. https://doi.org/10.1016/j.lindif.2012.09.013

Lee, V. (2013). The quantified self (QS) movement and some emerging opportunities for the educational technology field. Instructional Technology and Learning Sciences Faculty Publications, 480, 39-42. Recuperado de https://bit.ly/2KOJq5k

Liaw, S., \& Huang, H. (2013). Perceived satisfaction, perceived usefulness and interactive learning environments as predictors to self-regulation in e-learning environments. Computers \& Education, 60(1), 14-24. https://doi.org/10.1016/j.compedu.2012.07.015

López-Vargas, O., Ibáñez-Ibáñez, J., \& Racines-Prada, O. (2017). Students' metacognition and cognitive style and their effect on cognitive load and learning achievement. Journal of educational technology \& society, 20(3), 145-157. Recuperado de https://bit.ly/2KZzt5f

Olowookere, E., Alao, A., Odukoya, A., Adekeye, O., \& Agbude, G. (2015). Time management practices, character development and academic performance among University undergraduates: Covenant University experience. Creative Education, 6(1), 79-86. https://doi.org/10.4236/ce.2015.61007

Panadero, E. (2017). A review of self-regulated learning: six models and four directions for research. Frontiers in psychology, 8(422). https://doi.org/10.3389/fpsyg.2017.00422

Panadero, E., \& Alonso-Tapia, J. (2014). How do students self-regulate? Review of Zimmerman's cyclical model of self-regulated learning. Anales de Psicología, 30(2), 450-462. https://doi.org/10.6018/analesps.30.2.167221

Parra, J., Cerda, C., López-Vargas, O., \& Saiz, J. (2014). Género, autodirección del aprendizaje y desempeño académico en estudiantes de pedagogía. Educación y educadores, 17(1), 91-107. https://doi.org/10.5294/edu.2014.17.1.5

Pintrich, P. (1995). Understanding self regulated learning. New directions for teaching and learning, 1995(63), 3-12. https://doi.org/10.1002/tl.37219956304

Autorregulación del aprendizaje en la Formación Profesional a Distancia: efectos de la gestión del tiempo Cristian Jorge García-Marcos, Omar López-Vargas y Julio Cabero Almenara. Página 18 de 21 
RED. Revista de Educación a Distancia. Núm. 62, Vol. 20. Artíc. 06, 31-03-2020

DOI: http://dx.doi.org/10.6018/red.400071

Pintrich, P. (2004). A conceptual framework for assessing motivation and self-regulated learning in college students. Educational psychology review, 16(4), 385-407. https://doi.org/10.1007/s10648-004-0006-x

Rivera-Pelayo, V., Zacharias, V., Müller, L. \& Braun, S. (2012). Applying Quantified Self approaches to support reflective learning. En S. Dawson \& C. Hathornwaite (eds.), $L A K$ ' 12 Proceedings of the 2nd International Conference on Learning Analytics and Knowledge (p. 111-114). New York: ACM.

Rogers, J., \& Mirra, N. (2014). It's about time: Learning time and educational opportunity in California High Schools. Los Angeles: UCLA IDEA.

Rosário, P., Pereira, A., Högemann, J., Nunes, A., Figueiredo, M., Núñez, J., Fuentes, S., \& Gaeta, M. (2014). Self regulated learning: A systematic review based in scielo journals. $\begin{array}{lll}\text { Universitas } & \text { Psychologica, } & \text { 781-797. }\end{array}$ https://doi.org/10.11144/Javeriana.UPSY13-2.aars

Rossing, J., Miller, W., Cecil, A., \& Stamper, S. (2012). iLearning: The future of higher education? Student perceptions on learning with mobile tablets. Journal of the Scholarship of Teaching and Learning, 12(2), 1-26. Recuperado de https://bit.ly/2RktF7x

Rowe, F., \& Rafferty, J. (2013). Instructional design interventions for supporting selfregulated learning: enhancing academic outcomes in postsecondary e-learning environments. Journal of Online Learning and Teaching, 9(4), 590-601. Recuperado de https://bit.ly/2XdsGva

Schunk, D. H., \& Zimmerman, B. J. (eds.). (2012). Motivation and self-regulated learning: Theory, research, and applications. New York: Routledge.

Schunk, D. H., \& Zimmerman, B. J. (2013). Self-regulation and learning. En W. M. Reynolds \& G. E. Miller (eds.), Handbook of psychology. Educational psychology (7a ed., pp. 45-68). Hoboken, NJ: Wiley.

Sharon, T. (2017). Self-tracking for health and the quantified self: Re-articulating autonomy, solidarity, and authenticity in an age of personalized healthcare. Philosophy \& Technology, 30(1), 93-121. https://doi.org/10.1007/s13347-016-0215-5

Shin, D. H., \& Biocca, F. (2017). Health experience model of personal informatics: The case of a quantified self. Computers in Human Behavior, 69, 62-74. https://doi.org/10.1016/j.chb.2016.12.019

Autorregulación del aprendizaje en la Formación Profesional a Distancia: efectos de la gestión del tiempo Cristian Jorge García-Marcos, Omar López-Vargas y Julio Cabero Almenara.

Página 19 de 21 
RED. Revista de Educación a Distancia. Núm. 62, Vol. 20. Artíc. 06, 31-03-2020

DOI: http://dx.doi.org/10.6018/red.400071

Studenska, A. (2011). Educational level, gender and foreign language learning selfregulation difficulty. Procedia-social and behavioral sciences, 29, 1349-1358. https://doi.org/10.1016/j.sbspro.2011.11.373

Tseng, W. T., Liu, H., \& Nix, J. M. L. (2017). Self-regulation in language learning: Scale validation and gender effects. Perceptual and motor skills, 124(2), 531-548. https://doi.org/10.1177/0031512516684293

Tucker, R. (2014). Sex does not matter: gender bias and gender differences in peer assessments of contributions to group work. Assessment \& evaluation in Higher Education, 39(3), 293-309. https://doi.org/10.1080/02602938.2013.830282

Valencia-Vallejo, N., López-Vargas, O., \& Sanabria-Rodríguez, L. (2018). Effect of a motivational scaffolding on e-learning environments: self-efficacy, learning achievement, and cognitive style. Journal of educators online, 15(1), 1-14. Recuperado de https://bit.ly/2MSBFOu

Van Laer, S. \& Elen, J. (2017). In search of attributes that support self-regulation in blended learning environments. Education and Information Technologies, 22(4), 13951454. https://doi.org/10.1007/s10639-016-9505-x

Vohs, K., \& Baumeister, R. (2016). Handbook of self-regulation: Research, theory, and applications. New York: Guilford Publications.

Winne, P. (2016). Self-regulated learning. SFU Educational Review, 9. https://doi.org/10.21810/sfuer.v9i.300

Wolters, C. A. (2011). Regulation of motivation: Contextual and social aspects. Teachers College Record, 113(2), 265-283. Recuperado de https://bit.ly/2wWikkn

Yousaf, O., Popat, A., \& Hunter, M. (2015). An investigation of masculinity attitudes, gender, and attitudes toward psychological help-seeking. Psychology of men \& masculinity, 16(2), 234-237. https://doi.org/10.1037/a0036241

Yuselturk, E. \& Bulut, S. (2009). Gender differences in self-regulated online learning environment. Educational Technology \& Society, 12(3), 12-22. Recuperado de https://bit.ly/2wUMnJs

Zimmerman, B. J. (2008). Investigating self-regulation and motivation: Historical background, methodological developments, and future prospects. American

Autorregulación del aprendizaje en la Formación Profesional a Distancia: efectos de la gestión del tiempo Cristian Jorge García-Marcos, Omar López-Vargas y Julio Cabero Almenara.

Página 20 de 21 
RED. Revista de Educación a Distancia. Núm. 62, Vol. 20. Artíc. 06, 31-03-2020

DOI: http://dx.doi.org/10.6018/red.400071

educational research journal, 45(1), 166-183. https://doi.org/10.3102/0002831207312909

Zimmerman, B. J. (2013). From cognitive modeling to self-regulation: A social cognitive $\begin{array}{lll}\text { career path. Educational } \quad \text { psychologist, } & \text { 48(3), }\end{array}$ https://doi.org/10.1080/00461520.2013.794676

Zimmerman, B. J., \& Schunk, D. H. (Eds.). (2011). Educational psychology handbook series. Handbook of self-regulation of learning and performance. New York, NY, US: Routledge/Taylor \& Francis Group.

Autorregulación del aprendizaje en la Formación Profesional a Distancia: efectos de la gestión del tiempo Cristian Jorge García-Marcos, Omar López-Vargas y Julio Cabero Almenara. 